\title{
Mucosal Melanoma of the Head and Neck: Tata Memorial Hospital Experience
}

\author{
${ }^{1}$ Tejpal Gupta, ${ }^{2}$ Jai Prakash Agarwal, ${ }^{3}$ Suruchi Singh, ${ }^{4}$ Sarbani Ghosh-Laskar, ${ }^{5}$ Pankaj Chaturvedi \\ ${ }^{6}$ Shubhda Kane, ${ }^{7}$ Ashwini Budrukkar, ${ }^{8}$ Vedang Murthy, ${ }^{9}$ Anil K D'Cruz \\ ${ }^{1,4,7}$ Associate Professor, Department of Radiation Oncology, Tata Memorial Hospital, Parel, Mumbai, Maharashtra, India \\ ${ }^{2}$ Additional Professor, Department of Radiation Oncology, Tata Memorial Hospital, Parel, Mumbai, Maharashtra, India \\ ${ }^{3}$ Senior Registrar, Department of Radiation Oncology, Tata Memorial Hospital, Parel, Mumbai, Maharashtra, India \\ ${ }^{5}$ Associate Professor, Department of Head and Neck Surgical Oncology, Tata Memorial Hospital, Parel, Mumbai, Maharashtra, India \\ ${ }^{6}$ Professor, Department of Pathology, Tata Memorial Hospital, Parel, Mumbai, Maharashtra, India \\ ${ }^{8}$ Assistant Professor, Department of Radiation Oncology, Tata Memorial Hospital, Parel, Mumbai, Maharashtra, India \\ ${ }^{9}$ Professor, Department of Head and Neck Surgical Oncology, Tata Memorial Hospital, Parel, Mumbai, Maharashtra, India
}

Correspondence: Tejpal Gupta, Associate Professor, Department of Radiation Oncology, Tata Memorial Hospital, Dr Ernest Borges Road, Parel, Mumbai-400012, Maharashtra, India, Phone: 91-22-24177164, Fax: 91-22-24146937 e-mail: tejpalgupta@rediffmail.com

\begin{abstract}
Background: Primary mucosal melanoma of the head and neck (MMHN) is a rare, aggressive tumor of neural-crest origin. Despite universal progress in cancer care, the prognosis of $\mathrm{MMHN}$ continues to remain dismal.

Aims: To analyze and report the outcomes of primary head and neck mucosal melanomas treated at Tata Memorial Hospital.

Methods: Retrospective chart review of all patients with a diagnosis of nonocular MMHN presenting to the institute between 1995 to 2003. Locoregional control and disease-free survival were used as outcome measures.

Results: 42 patients presenting within the study period with nonocular MMHN (oral-55\%, sinonasal- $40 \%$, and pharyngeal-5\%) at a median age of 53 years constituted the demographic cohort. 11 (26\%) patients not amenable to any active anticancer treatment were treated with best supportive care alone and excluded from outcome analysis. 26 patients underwent surgery with complete resection of tumor. Seven $(27 \%)$ also received adjuvant radiotherapy due to the adverse histopathologic features. Two patients were treated with radical radiotherapy due to unresectability, two patients received palliative chemotherapy, while one patient was treated with definitive chemoradiotherapy. With a mean follow-up of 11 months (range 1-58 months), the 3-year locoregional control and disease-free survival was $41 \%$ and $12 \%$ respectively. Age, sex, site of primary, tumor stage, surgical resection, margin status, depth of infiltration, and adjuvant radiotherapy did not affect outcome significantly.

Conclusion: Primary mucosal melanoma of the head and neck is a rare, but, aggressive tumor with a dismal prognosis. Surgical resection with clear margins offers the best chance of cure for early localized disease. The high incidence of locoregional as well as distant failures after surgical resection supports the use of adjuvant therapy. Deeper insights into the pathobiology of disease can help develop more specific and effective treatment strategies to improve long-term outcomes.
\end{abstract}

Keywords: Mucosal melanoma, Sinonasal, Oral, Pharyngeal, Management, Prognosis.

\section{BACKGROUND}

Primary mucosal melanoma of the head and neck (MMHN) is a rare, but aggressive tumor comprising $<1 \%$ of all melanomas. ${ }^{1,2}$ It originates from melanocytes that have migrated as neuroectodermal derivatives in the mucosa of the upper aerodigestive tract with a predilection for sinonasal, oral, and pharyngeal in accordance with their neuralcrest origin. ${ }^{2}$ The relative rarity of this disease has precluded the conduct of prospective clinical trials for evidence-based decision making. Nevertheless, it is well recognized that surgical excision with adequate margins offers the best chance for local control of localized MMHN. ${ }^{3,4}$ However, in mucosal melanomas originating in the head and neck region, surgery is often limited by anatomic, cosmetic, and functional considerations. In this context, postoperative adjuvant radiotherapy has been variably used for maximizing locoregional control and improving survival. ${ }^{5}$ The role of chemoimmunotherapy in localized MMHN is still not very well defined and continues to evolve. In contrast to well- 
established etiologic factors and reliable prognostic factors for cutaneous melanomas, such information is lacking for mucosal melanomas, including MMHN. Despite better understanding of the pathobiology of disease, refinements and advancements in surgical techniques, frequent use of high-precision radiotherapy, including heavy ions, and availability of newer biochemotherapy regimens, the prognosis of this disease continues to remain dismal ${ }^{6}$ with very high rates of local failure and frequent appearance of distant metastases resulting in consistently poor long-term survival (10-25\%).

\section{AIMS}

This retrospective study aimed to analyze and report the outcomes of primary head and neck mucosal melanomas treated at Tata Memorial Hospital. It also attempted to identify prognostic and therapeutic factors that could potentially impact upon outcome (locoregional and disease free survival) in MMHN.

\section{MATERIALS AND METHODS}

The medical records of patients with a histopathological diagnosis of melanoma of the head and neck region presenting to the institute between 1995 and 2003 were reviewed retrospectively. Patients with cutaneous melanoma of the head and neck, ocular or uveal melanomas were excluded restricting only to head and neck mucosal melanomas. Tumor extent was evaluated by physical examination, examination under anesthesia, and appropriate imaging. All patients were retrospectively staged using the 2002 TNM system for head and neck cancers. Pertinent patient and treatment data were extracted from retrospective chart review. All patients were required to follow-up initially at 4-6 weeks from the completion of therapy and subsequently at 3-6 monthly intervals for the first two years and annually thereafter. All efforts were made to contact patients or their relatives telephonically to update their disease status. Patients not responding to above measures were considered lost to follow-up and censored for statistical consideration. Locoregional control and disease-free survival were used as outcome measures and calculated using the Kaplan-Meier method. Short duration of followup precluded estimation of overall survival. All estimates were calculated from the date of initiation of therapy till the defined event if any or until last contact or death. All statistical analysis was done on SPSS version 14.0.

\section{RESULTS}

Between 1995 and 2003, 42 patients presenting to the institute were diagnosed to have a mucosal melanoma of the head and neck and constitute the study cohort. Their median age at diagnosis was 53 years (range 22-75 years). There were 27 males and 15 females in this cohort. 23 (55\%) patients had primary disease in the oral cavity, 17 (40\%) in the sinonasal tract, and 2 (5\%) in the pharynx. Eleven of these 42 (26\%) patients presenting with loco-regionally advanced or metastatic disease and/or poor performance status not amenable to any active anticancer treatment were treated with best supportive care alone. They were excluded from further analysis, leaving 31 patients treated with definite oncological treatment eligible for analysis of outcome measures. Twenty-six patients underwent surgery with the complete resection of tumor. Sixteen patients also underwent neck dissection either electively for the clinically negative (N0) neck or therapeutically for the clinically positive neck $\left(\mathrm{N}^{+}\right)$, of which 5 were bilateral. Twelve of the ipsilateral neck dissections were positive for nodal metastases whereas only one of the contralateral neck dissections showed pathological evidence of disease. Seven of the 26 patients (27\%) undergoing surgery also received postoperative adjuvant radiotherapy due to the presence of any one of the following factors, viz. close or positive margins, single positive lymph node with perinodal extension, multiple nodes, deep muscle and soft tissue infiltration. Two patients were treated with radical radiotherapy due to unresectability, two patients received chemotherapy as palliative treatment, and one patient with pharyngeal melanoma received chemoradiotherapy as primary treatment. Radiotherapy was delivered either with conventional fractionation (200 cGy/fraction) or modestly high dose per fraction (225-300 cGy/fraction) with megavoltage beams with shrinking fields to doses of 50-60 Gy conventional fractionation equivalent. With a mean follow-up of 11 months (range 1-58 months), the 3-year locoregional control and disease-free survival was $41 \%$ and $12 \%$ respectively for the 31 patients treated with any active anticancer treatment (Figs 1A and B). The median diseasefree survival was 11 months only (95\% confidence interval 7.6-14.3 months). Locoregional failures were seen in 13 patients (including 3 with persistent primary disease, 3 with isolated local recurrence, and 2 with isolated nodal relapse); while distant metastases were seen in 8 patients ( 5 isolated and 3 with some local or regional component) (Fig. 2). The 

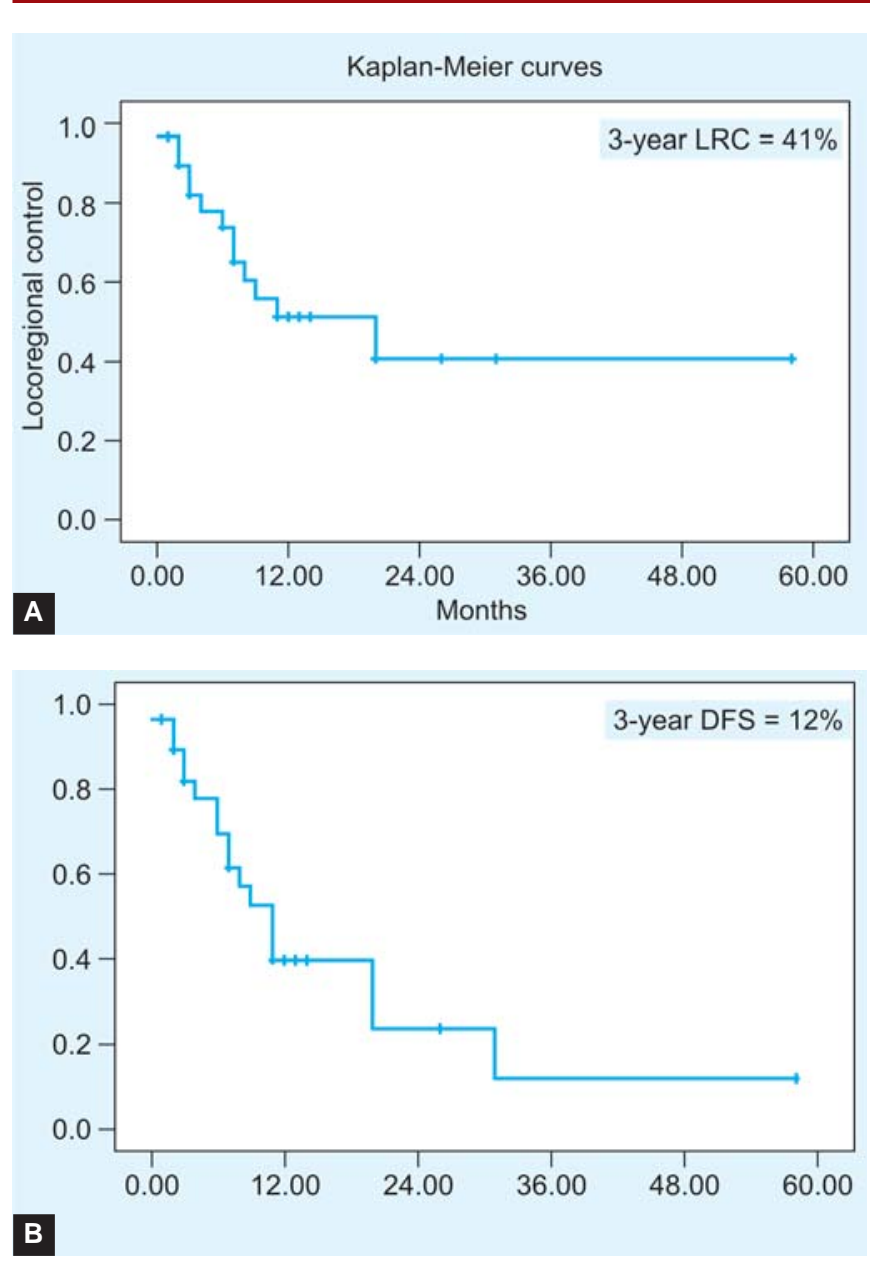

Figs $1 \mathrm{~A}$ and $\mathrm{B}$ : 3-year Kaplan-Meier estimate of $(\mathrm{A})$ locoregional control and $(B)$ disease-free survival

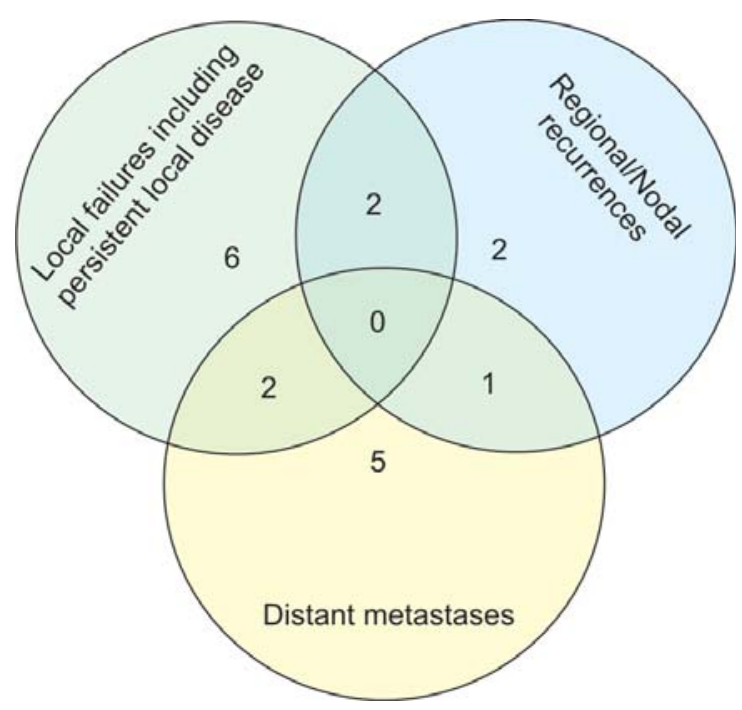

Fig. 2: Patterns of failure in mucosal melanomas of the head and neck impact of various patient and treatment factors on outcome was analyzed. Age, sex, site of primary, tumor stage, surgical resection, margin status, and depth of infiltration did not affect outcome significantly. The use of adjuvant radiotherapy also did not improve locoregional control or disease-free survival. Analysis of complications was not done due to inadequate information on treatment related toxicity in the medical charts.

\section{DISCUSSION}

Primary mucosal melanoma of the head and neck is a rare, but aggressive tumor comprising $<1 \%$ of all melanomas. ${ }^{1,2}$ It originates from melanocytes that have migrated as neuroectodermal derivatives in the mucosa of the upper aerodigestive tract with a predilection for sinonasal, oral, and pharyngeal mucosa in accordance with their neural-crest origin. ${ }^{2}$ Amongst MMHN, over half occur in the sinonasal region (56\%) followed by oral cavity (40\%) and laryngopharynx (4\%). Oral mucosal melanomas occur predominantly over the hard and soft palate (42\%) and alveolar gingival (28\%) with other sub-sites, such as buccal mucosa, lip, tongue and floor of mouth making up the remainder. $^{7}$ Oral-pharyngeal mucosal melanomas tend to have worse outcomes compared to sinonasal melanomas, ${ }^{8,9}$ possibly due to higher chances of lymph node involvement and hematogenosus dissemination. In contrast to cutaneous melanomas, those involving the mucosal surfaces typically present at a more aggressive vertical growth (nodular) phase with deep invasion of underlying submucosa. Majority of them do not exhibit radial (superficial spreading) growth phase. ${ }^{10,11}$ Since they do not fit into any specific cutaneous melanoma category, attempts have been made to arrive at a standardized nomenclature for this unique oral malignancy. ${ }^{12}$ Several histopathological parameters including depth of invasion ${ }^{13}$ have a strong predictive value in determining recurrence and survival in patients with cutaneous melanoma. Such standardized correlation of histopathological features with outcome is lacking in mucosal melanomas. In a retrospective multicentric study ${ }^{14}$ involving 115 patients with mucosal melanoma; age $<50$ years, cut margin negativity, genitourinary primary site, and lower nodal burden were found to be favorable prognostic factors for survival. A prognostic model based on age, resection status, primary site, and lymph node status was proposed from a subset analysis of 87 patients who underwent curative resection (R0 or R1) that robustly separated patients into 
three risk groups with significantly different survival outcomes.

It is often difficult to define the optimal treatment modality in MMHN due to the small number of patients in most studies, retrospective design, and inherent selection bias. ${ }^{15}$ Nevertheless, it is generally accepted that surgical resection with clear margins results in maximal locoregional control and offers the best chance of cure. ${ }^{3,4,16}$ The efficacy of radiotherapy as primary definitive treatment has not been adequately tested, as patients with small, resectable tumors undergo upfront surgery reserving primary radiotherapy for advanced, unresectable disease. However, in a recent report, ${ }^{17} 30$ patients were treated with definitive primary radiotherapy while 25 patients were treated with surgery with or without postoperative adjuvant radiotherapy. The 5 -year overall and cancer-specific survival was $22 \%$ and $32 \%$ respectively. There were no significant differences in outcomes between the two groups suggesting a contrarian view that primary definitive radiotherapy may be worthy of consideration in the curative treatment of MMHN. The use of postoperative adjuvant radiotherapy in HNMM has steadily increased over the years, however its role in improving outcomes still remains controversial. ${ }^{5}$ While it has been associated with improved locoregional control in several retrospective series, ${ }^{18-25}$ this has never translated into consistent, meaningful survival benefit. Experimental radiobiological data shows large variations in radiosensitivity of human melanomas, which are dominated by the intrinsic radio-resistance and high capacity for sublethal damage repair. ${ }^{26}$ Due to inherent radioresistance, it has been postulated that hypofractionation, ${ }^{27}$ i.e high-dose per fraction (>3 Gy) or high linear energy transfer (LET) radiation ${ }^{28}$ could improve outcomes in mucosal melanomas. However, the optimal radiotherapy schedule still remains uncertain. The role of biochemotherapy in localized MMHN is not well defined and continues to evolve. ${ }^{29}$ Nevertheless, there is emerging evidence that such systemic approaches can lead to impressive response rates in advanced or metastatic mucosal melanoma that can potentially improve survival. ${ }^{30}$

This study however failed to demonstrate the impact of surgical resection upon outcome. Even, the use of adjuvant radiotherapy did not improve locoregional control or survival compared to surgery alone. The reasons for this could be the small number of patients, advanced stage of disease at presentation, and bias of treating more extensive- stage disease with combined-modality treatment. Only a small subset (27\%) of patients received postoperative adjuvant radiotherapy, which was delivered with megavoltage photon beams as conventional fractionation and not utilizing hypofractionated regimens or high-LET radiation. The development of distant metastases is a serious problem associated with MMHN. Despite very high rates (51-68\%) of distant metastases, systemic chemotherapy and/ or immunotherapy has yielded disappointing results both in the adjuvant and palliative setting. One-third of patients in the present study developed distant metastases which is somewhat lesser than previously published literature. This may be partly explained by relatively short duration of follow-up and the prevalent institutional practice of performing imaging evaluation of distant metastases only in symptomatic patients.

\section{CONCLUSION}

Primary mucosal melanoma of the head and neck is a rare, but aggressive tumor with a relatively poor prognosis. Despite universal progress in cancer care, the outlook for MMHN has not changed and remains dismal even today. Surgical resection with clear margins offers the best chance of cure for early localized disease. The high incidence of locoregional as well as distant failures after surgical resection supports the use of adjuvant therapy. Future efforts should focus on the optimization of radiotherapy to maximize locoregional control and integration of novel biochemotherapy regimens to enhance systemic control. Deeper insights into the pathobiology of disease can help develop more specific and effective treatment strategies, including molecularly targeted therapy to improve long-term outcomes.

\section{REFERENCES}

1. Chang AE, Karnell LH, Menck HR, et al. The National Cancer Data Base report on cutaneous and noncutaneous melanoma: A summary of 84,836 cases from the past decade. The American College of Surgeons Commission on Cancer and the American Cancer Society. Cancer 1998;83(8):1664-78.

2. Manolidis S, Donald PJ. Malignant mucosal melanoma of the head and neck. Review of literature and report of 14 patients. Cancer 1997;80:1373-86.

3. Lee SP, Shimizu KT, Tran LM, et al. Mucosal melanoma of the head and neck: The impact of local control on survival. Laryngoscope 1994;104:121-26.

4. Mendenhall WM, Amdur RJ, Hinerman RW, et al. Optimal treatment and outcomes for head and neck mucosal melanoma. Am J Clin Oncol 2005;28(6):626-30. 
5. Krengli M, Jereczek-Fossa BA, Kaanders JHAM, et al. What is the role of radiotherapy in the treatment of mucosal melanoma of the head and neck? Crit Rev Oncol Hematol 2008;65: 121-28.

6. Moreno MA, Hanna EY. Management of mucosal melanomas of the head and neck: Did we make any progress. Curr Opin Otolaryngol Head Neck Surg 2010;18:101-06.

7. Hicks MJ, Flaitz CM: Oral mucosal melanoma: Epidemiology and pathobiology. Oral Oncol 2000;36:152-69.

8. McLean M, Tighiouart M, Muller S. Primary mucosal melanoma of the head and neck. Comparison of clinical presentation and histopathologic features of oral and sinonasal melanoma. Oral Oncol 2008;44:1039-46.

9. Moreno MA, Roberts DB, Kupferman ME, et al. Mucosal melanoma of the nose and paranasal sinus: A contemporary experience from the MD. Anderson Cancer Centre. Cancer 2010;116:2215-23.

10. Breslow A. Thickness, cross-sectional areas and depth of invasion in the prognosis of cutaneous melanoma. Ann Surg 1970;172(5):902-08.

11. Batsakis JG, Suarez P, El-Naggar AK, et al. Mucosal melanomas of the head and neck. Ann Otol Rhinol Laryngol 1998;107: 626-30.

12. Barker BF, Carpenter WM, Daniels TE, et al. Oral mucosal melanomas: The WESTOP Banff workshop proceedings. Western Society of Teachers of Oral Pathology. Oral Radiol Endod 1997;83:672-79.

13. Mizoe JE, Tsujii H, Kamada T, et al. MRI of mucosal malignant melanoma of the head and neck. Int J Radiat Oncol Biol Phys 2004;60(2):358-64.

14. Kim HS, Kim EK, Jun HJ, et al. Noncutaneous malignant melanoma: A prognostic model from a retrospective multicentre study. BMC Cancer 2010;10:167.

15. Bachar G, Loh KS, O’Sullivan B, et al. Mucosal melanomas of the head and neck: The Princess Margaret Hospital experience. Head Neck 2008;30:1325-31.

16. Medina JE, Ferlito A, Pellitteri PK, et al. Current management of mucosal melanoma of the head and neck. J Surg Oncol 2003;83:116-22.

17. Douglas CM, Malik T, Swindell R, et al. Mucosal melanoma of the head and neck: Radiotherapy or surgery? J Otolaryngol Head Neck Surg 2010;39:385-92.
18. Nandapalan V, Roland NJ, Helliwell TR, et al. Mucosal melanoma of the head and neck. Clin Otolaryngol 1998;23: 107-16.

19. Patel SG, Prasad ML, Escrig M, et al. Primary mucosal malignant melanoma of the head and neck. Head Neck 2002;24:247-57.

20. Wada H, Nemoto K, Ogawa Y, et al. A multi-institutional retrospective analysis of external radiotherapy for mucosal melanoma of the head and neck in Northern Japan. Int J Radiat Oncol Biol Phys 2004;59(2):495-500.

21. Temam S, Mamelle G, Marandas P, et al. Postoperative radiotherapy for primary mucosal melanoma of the head and neck. Cancer 2005;103:313-19.

22. Krengli M, Masini L, Kaanders JHAM, et al. Radiotherapy in the treatment of mucosal melanoma of the upper aerodigestive tract: Analysis of 74 cases. A Rare Cancer Network study. Int J Radiat Oncol Biol Phys 2006;65(3):751-59.

23. Nakashima JP, Viegas CM, Fassizoli AL, et al. Postoperative adjuvant radiation therapy in the treatment of primary head and neck mucosal melanomas. ORL 2008;70:344-51.

24. Meleti M, Leemans CR, de Bree R, et al. Head and neck mucosal melanoma: Experience with 42 patients with special emphasis on the role of postoperative radiotherapy. Head-Neck 2008;30:1543-51.

25. Wu AJ, Gomez J, Zhung JE, et al. Radiotherapy after surgical resection for head and neck mucosal melanoma. Am J Clin Oncol 2010;33:281-85.

26. Dewey L. The radiosensitivity of melanoma cells in culture. $\mathrm{Br}$ J Radiol 1971;44:816-17.

27. Bentzen S, Overgaard J, Thames HD, et al. Clinical radiobiology of malignant melanoma. Radiother Oncol 1989;16:169-82.

28. Yanagi T, Mizoe JE, Hasegawa A, et al. Mucosal melanoma of the head and neck treated with carbon ion radiotherapy. Int J Radiat Oncol Biol Phys 2009;74:15-20.

29. Palma MS, Muthukumaran S, Stephanie FB, et al. Adjuvant immunotherapy for patients with melanoma: Are patients with melanoma of the head and neck candidates for this therapy? Head-Neck 1997; 19:595-603.

30. Bartell HL, Bedikian AY, Papadopopoulos NE, et al. Biochemotherapy in patients with advanced head and neck mucosal melanoma. Head-Neck 2008;30;1592-98. 